TEMAS DE ACTUALIDAD

Rev Chil Salud Pública 2018; Vol 22 (1): 71-74

\title{
DE LA SALUD PÚBLICA A LA SALUD \\ COLECTIVA DESDE EL PARADIGMA DE LA COMPLEJIDAD. DESAFÍO PARA LA GESTIÓN PÚBLICA VENEZOLANA
}

FROM PUBLIC HEALTH TO COLLECTIVE HEALTH, THROUGH THE

COMPLEXITY PARADIGM. CHALLENGES FOR VENEZUELAN PUBLIC

MANAGEMENT

\section{INTRODUCCIÓN}

Cada vez se evidencia la necesidad de transcender aspectos relacionados que interfieren con el desarrollo eficaz y eficiente de las gestiones públicas; tal como es el caso de la escasa participación de la comunidad de las instituciones científicas y de las académicas, en la elaboración de los programas en salud pública, que pueden brindar un valioso aporte para los problemas que aquejan a la comunidad. Limitaciones como éstas son un factor preponderante que conllevan en reiteradas ocasiones al fracaso de las gestiones públicas, lo que incide desfavorablemente en la población.

Es axiomático la importancia de un cambio de paradigma, un nuevo enfoque de las políticas públicas, donde se tomen en consideración las opiniones de todos los sectores que conforman la sociedad y desde la praxis se desarrollen las ideas, acciones y decisiones que ayuden a concebir una salud colectiva que contribuya al mejoramiento y cuidado de la salud de la población sin obviar el entorno en el que la comunidad se desenvuelve, y su medio ambiente, a la vez que involucrar todos los aspectos socioantropológicos que caracterizan a la comunidad, como por ejemplo la cultura, la religión y los estilos de vida.

Es pertinente y necesario la creación de espacios de formación y de reflexión destinados a utilizar nuevos enfoques desde el pensamiento complejo, para estudiar no solo la actividad gubernamental, sino la revisión de las políticas públicas y evaluar en conjunto con todos los actores sociales si cumplen o no con las expectativas para un buen vivir. Aunado a ello, es importante develar donde están las fallas que retardan o frenan el desarrollo de la salud colectiva como alternativa para brindar las respuestas satisfactorias que todos los ciudadanos se merecen, para vivir con dignidad y calidad de vida.

Laboratorio Clínico, Servicio Médico,

Instituto Venezolano de Investigaciones Científicas. mayralopezcaldea@gmail.com 


\section{Una perspectiva socioantropológica para la construcción de las políticas en salud publica}

En palabras de Gadamer, "La nueva salud pública está en la obligación de nutrirse de diferentes aportes teórico-metodológicos que aborden la salud y la enfermedad, desde un pensamiento que equilibre el orden y el caos, con las bifurcaciones y con las subjetividades. Debe estar en constante construcción de conocimientos en salud desde una visión estratégica situacional. Debe constituir un proceso de encuentro, un genuino desarrollo cognoscitivo, una hermeneusis que se aproxime al ser y a sus circunstancias" $(1984$, p. 35$)$.

Desde esta perspectiva, la salud pública debe estar en frecuente revisión teórica y práctica, debe adaptarse a los cambios y momentos históricos que vive la sociedad y en esta era de globalización -acompañada de transformaciones ecológicas, transculturación, movimientos sociales, industrialización, consumismo y medicalización exacerbada-, amerita la construcción de una nueva salud que tome en consideración todos y cada uno de estos aspectos aunados a los factores sociales, precisamente lo que plantea la salud colectiva.

En este orden de ideas, Sosa (2006) expresa que "la salud colectiva pretende centrar su enfoque en la construcción desde la participación de los colectivos poblacionales y de los movimientos sociales organizados, en la construcción del sujeto actor colectivo, constructor de la salud y la vida comunitaria. Esto incluye directamente al buen vivir como base de la salud colectiva”. (p. 193).

En virtud de la multidisciplinariedad a la que se puede integrar la socioantropología a la salud colectiva, surge como subdisciplina la antropología médica, que entiende "la salud en el ámbito de la cultura y comprende el trinomio salud-enfermedad-atención, como esencial, para que cada comunidad humana desarrolle una respuesta específica, lo que le otorga suficiente variabilidad. Estudia las dimensiones históricas, biológicas, psicológicas, culturales y sociales, que determinan el modo en que las personas entienden y viven la salud y sus problemas de salud. Tiene como objeto de estudio y análisis los distintos sistemas, creencias y prácticas respecto a la salud y al proceso salud-enfermedad-atención en cualquier tipo de sociedad" (Pizza 2013, p. 267). De allí la importancia de considerar a la salud como un espacio socioantropológico, con una mirada transdisciplinaria y compleja, donde todos los sectores deben estar involucrados en la participación para el diseño de las políticas públicas y su respectiva gestión.

En este sentido, la salud colectiva es un servicio público incluido en los derechos económicos sociales, culturales y ambientales en tanto sujetos de derechos y deberes. Es además una construcción política en la que necesariamente deben participar el Estado y la Sociedad civil asumiendo la interacción de múltiples sectores sociales y determinantes en la calidad de vida y la salud de las poblaciones. (Ruiz 2005, p. 58; Díaz, Aguilar y Linares 2015, p. 655).

\section{Construcción de la gestión pública en salud desde el paradigma de la complejidad}

Diseñar las políticas públicas en el sector salud de una nación desde el paradigma de la complejidad, es un proceso que involucra y también está muy asociado a definiciones institucionales y a las prácticas políticas propias de cada comunidad.

Parafraseando a Motta (1993), la participación gerencial es un proceso complejo que requiere de la intervención de actores sociales y de la eficacia de la administración del poder que recae sobre el gobierno de turno. (p. 95). Es por ello, que pensar desde la complejidad en salud colectiva, permite percibir la realidad como un sistema complejo-abierto, con diferentes niveles organizativos, donde cada nivel, corresponden a cualidades emergentes, que necesitan de estrategias que responda a diferentes saberes y perspectivas.

En este sentido, Morin (1990), expresa que "La complejidad no significa complicación, por el contrario, vincula multiplicidad de factores que se entrelazan sin intentar dar demostraciones casualistas o finalistas. Es un 
pensamiento articulante y multidimensional" (p. 33). Es indispensable contribuir a sustentar la noción de salud incluyendo como prioridad las estrategias de intervención poblacional desde una mirada compleja, para ello es necesario transcender el pensamiento simple disyuntivo a una racionalidad compleja con intervención de varias disciplinas y la participación de los actores/as sociales.

Es indudable que la globalización ha contribuido a transformar las realidades y de ello no escapan los modelos de gestión pública, donde dichos modelos deben ser diseñados desde la transdiciplinariedad, con la integración de diversos saberes, desde el conocimiento popular de las comunidades, con la participación de las comunidades científicas y académicas, para la construcción de una nueva salud pública que transcienda hacia una salud colectiva con el enfoque epistemológico de la complejidad.

De allí la importancia que los modelos de gestión pública generen cambios que fortalezcan de manera sustentable y sostenible a la población y su medio ambiente. Este modelo se debe iniciar con los estudiantes de pregrado, pero no solo del sector salud, sino que se haga extensivo a todas las profesiones in complexus e inclusive a otras áreas del conocimiento y conformar equipos transdisciplinario, integrados esencialmente por la participación ciudadana que vive y padece los problemas que los aquejan, para que aporten ideas, innovaciones y soluciones al buen vivir.

\section{La participación ciudadana en la gestión pública en salud}

La participación ciudadana en salud, tiene una gran variedad de características que van en pro de la construcción de la salud con la participación de la comunidad. Este enfoque rechaza las concepciones y características del modelo medico hegemónico y por el contrario asume la salud como un derecho social y humano cuya responsabilidad de la salud ciudadana es del Estado pero sin excluir del todo la corresponsabilidad individual; por lo tanto, la salud es un bien público que debe estar enfocada en el bienestar y calidad de vida con visión holística, integral, en cuyo fundamento primordial deben estar implícitos los aspectos socioantropológicos, reivindicando el saber popular, la cultura, la religión, la tradición, la historia, en todo el proceso de salud-enfermedad. (Uzcátegui 2014, p. 65).

En Venezuela, el marco legal normativo de las políticas que orientan la salud indican cuáles son los lineamientos de las acciones hacia la planificación de la atención sanitaria; en ellas se expresan los principios orientadores, siendo uno de ellos la participación social para la solución de los problemas a través de la toma de decisiones que impactan sobre el desarrollo social y la salud, tal como lo promulga la Constitución de la República Bolivariana de Venezuela en sus artículos, 83 y 86, así como los lineamientos generales del Ministerio de Salud y Desarrollo Social. Pero además de ellos existen otras leyes, reglamentos, código y planes estratégicos que promueven la participación comunitaria a la toma de decisiones en las políticas públicas, como son la ley de participación ciudadana y protagónica en su artículo $3^{\circ}$, el Plan Estratégico de Salud y Desarrollo Social, el Plan de la Patria, la Ley Orgánica de la Administración Social, entre los más relevantes.

\section{CONCLUSIÓN}

Desde el paradigma de la complejidad, es interesante analizar en la actualidad cuáles han sido las experiencias en Venezuela, en lo que a servicio de salud se refiere y cuál ha sido la intervención de las comunidades y la participación ciudadana en este proceso, y así profundizar los conocimientos acerca de los modelos de gestión pública existentes en la nación y si éste redunda en el buen vivir de la comunidad.

De acuerdo con el marco legal venezolano, es evidente que se han diseñado leyes que han tomado en consideración la participación social como factor para asegurar el desarrollo humano integral y una existencia digna y provechosa para la población venezolana. No obstante, actualmente vivimos ante una crisis política, económica y social que influye en el modo de vivir de la población, manifestándo- 
se en un estilo de vida donde predomina el estrés social, la intolerancia, el abuso de poder, la escases de insumos en los centros asistenciales, inseguridad y otros aspectos que influyen en la calidad de vida del venezolano y por ende en su salud integral, quedando la ciudadanía subsumida ante todos estos factores con los que debe pugnar en la cotidianidad. En tal sentido, aun cuando existen leyes que propenden a la participación ciudadana, es mucho el camino por transitar para alcanzar una gestión púbica cuyos principios estén fusionados con los que plantea la salud colectiva, que tiene como fin único el bienestar y buen vivir para la comunidad.

\section{REFERENCIAS}

1. Díaz Z, Aguilar T, Linares X. La antropología médica aplicada a la salud pública. $R e-$ vista Cubana de Salud Pública, 2015; 41(4): 655-665

2. Gadamer H. Verdad y método. Fundamento de una hermenéutica filosófica. España: Sígueme, 1984; p. 35.

3. González MC, Sosa G. Aspectos epistemológicos de la salud pública. Aproximaciones para el debate. Salus, 2010; 14 (AbrilSin mes) [visitado 10.07.2017]. Disponible en: http://www.redalyc.org/articulo. oa? $\mathrm{id}=375939012010$
4. Motta PR. La ciencia y el arte de ser dirigente. Un instrumento indispensable para el desarrollo personal y profesional de actuales y futuros dirigentes. Bogotá: Tercer Mundo Editores, 1993; p. 95.

5. Morin E. Introducción al pensamiento complejo. Barcelona: Gedisa, 1990; p. 33.

6. Pizza G. Antropología Médica: una propuesta de investigación. En: Lisón Tolosana C. (Ed.). Introducción a la Antropología social y cultural. Teoría, método y práctica. España: Ediciones AKAL, 2013; pp. 267-287.

7. Ruiz IC. Salud en calidad de vida. En: Franco S. (Comp.) La salud pública hoy. Colombia: Universidad Nacional de Colombia, 2005; p. 58

8. Sosa S, G. Un Médico más Cercano al Hombre y a la Salud. La formación para la praxis desde una perspectiva sociocrítica. Capítulo V. Valencia, Venezuela: Clemente Editores, 2006; pp. 193-227-273.

9. Uzcátegui J. De la participación en salud a la construcción del poder popular: Experiencias para el debate. Maracay, Venezuela: IAES. Salud y participación popular: hacia una ciudadanía plena, 2014; p. 65. 